\title{
G

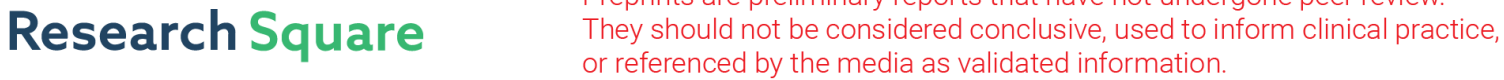 \\ Efficacy and Safety of Deep Acupuncture at Qugu Point (CV2) for Benign Prostatic Hyperplasia: Study Protocol for a Randomized Controlled Trial
}

\section{Han Tang}

China Academy of Chinese Medical Sciences Xiyuan Hospital

\section{Yonghui Lu( $\nabla$ yhlu2008@sina.com )}

China Academy of Chinese Medical Sciences Xiyuan Hospital https://orcid.org/0000-0002-3658-9908

\section{Hua Ren}

China Academy of Chinese Medical Sciences Xiyuan Hospital

Jianhua Fu

China Academy of Chinese Medical Sciences Xiyuan Hospital

\section{Yulian Meng}

China Academy of Chinese Medical Sciences Xiyuan Hospital

\section{Baoying Li}

China Academy of Chinese Medical Sciences Xiyuan Hospital

\section{Fang Lu}

China Academy of Chinese Medical Sciences Xiyuan Hospital

\section{Xinzi Song}

China Academy of Chinese Medical Sciences Xiyuan Hospital

\section{Research Article}

Keywords: Benign prostatic hyperplasia, Acupuncture, Qugu point, CV2, Prostate capsule, Lower urinary tract symptoms, Study protocol, Randomized controlled trial

Posted Date: November 16th, 2021

DOI: https://doi.org/10.21203/rs.3.rs-818097/v1

License: (c) (1) This work is licensed under a Creative Commons Attribution 4.0 International License. Read Full License 


\section{Abstract}

Background: Benign prostatic hyperplasia (BPH) is one of the most common urological disease and often associated with lower urinary tract symptoms (LUTS) in elderly men. Chinese acupuncturists found that deep acupuncture at Qugu point (CV2) can effectively relieve urination difficulties and improve quality of life of patients with troublesome BPH/LUTS. However, there are currently insufficient scientific evidence and well-designed trials to support its effect as well as safety in the treatment of BPH/LUTS. Therefore, this study protocol is designed to evaluate the effectiveness and safety of deep acupuncture at CV2 for men with BPH/LUTS.

Methods: A single-center, randomized, subject-and assessor-blinded, placebo-controlled trial will be conducted in an outpatient acupuncture department in Beijing, China. A total of 120 subjects (50-80 yr) will be randomized to one of three balanced groups: deep acupuncture group 1 (deep acupuncture at CV2 with needle tip reaching the prostate capsule), deep acupuncture group 2 (deep acupuncture at CV2 with needle tip $10 \mathrm{~mm}$ away from the prostate capsule), and sham acupuncture group (sham acupuncture with non-penetrating needle) in a ratio of 1:1:1. The study period for each subject will be 4 months, including 4 weeks baseline observation period, 4 weeks intervention period, and 8 weeks follow-up period. All subjects will receive 10 treatments during the intervention period. Subjects in deep acupuncture groups will be measured the needle depth and angle by computerized tomography (CT) in their first treatment. The primary outcome is the change in International Prostate Symptom Score. Secondary outcomes include quality of life, maximum urinary flow rate, and post-void residual.

Discussion: The objectives of this study include: 1) to assess the efficacy of deep acupuncture at CV2 in the treatment of $\mathrm{BPH} ; 2$ ) to evaluate the safety of acupuncture at CV2 by measure the needle depth and angle under $\mathrm{CT}$. This study will provide an evidence-based recommendation for acupuncture at CV2 in the treatment of $\mathrm{BPH}$.

Trial registration: Registered on 18 January 2020. Chinese Clinical Trials Register, ChiCTR2000029203.

\section{Introduction}

\section{Background and rationale $\{6 a\}$}

Benign prostatic hyperplasia (BPH) is a histological definition of smooth muscle and epithelial cells proliferating in prostatic transition zone[1]. The enlarged gland may compress the urethra and result in bladder outlet obstruction (BOO), which contribute to lower urinary tract symptoms (LUTS)-a common complaint with serious influence on quality of life in most elderly men[2,3]. LUTS secondary to BPH are clinically characterized by storage symptoms (urgency, frequency, nocturia and urge incontinence) and voiding symptoms (hesitancy, poor urinary flow, straining, prolonged urination, sensation of incomplete emptying)[4]. The prevalence of BPH rises with increasing age and the incidence rate $\sim 50 \%$ of men aged 51 to $60 \mathrm{yr}[5,6]$. In 1997, the total prevalence of BPH was found to be $43.68 \%$ according to a prevalence study based on 3,361 men $\nabla>60 \mathrm{yr} \otimes$ lived in six cities in China[7]. With an increasingly aging population, 
the incidences of BPH are increasing rapidly and causing a significant socioeconomic burden[8]. The management of $\mathrm{BPH}$ is complex. As not all men with $\mathrm{BPH}$ will be troubled by bothersome symptoms, formal assessment of patient's condition should be completed prior any intervention[2,9]. Watchful waiting is usually recommended for asymptomatic BPH. Medical therapy has been widely accepted as first-line treatment for men with moderate-to-severe $\mathrm{BPH}[10,11]$. Surgical is often suggested for men with poor efficacy of medications and with severe complications (e.g., renal insufficiency, refractory urinary retention, recurrent urinary tract infections)[12]. However, some patients still troubled by poor curative effect or side-effects of medications[13,14] or surgical treatments[15]. These patients are interested in explore more alternative and complementary approaches. Based on the principle of improving the quality of life of men with bothersome BPH/LUTS, alternative options need to be taken into condition.

Acupuncture has a good effect in the treatment of BPH for hundreds of years, and is widely used in China[16]. A sample of 33 patients in our pilot study shows that deep acupuncture at Qugu point (CV2) could effectively alleviate symptoms caused by BPH/LUTS[17]. The location of CV2 is in the lower abdomen, the upper edge of the pubic symphysis and the anterior midline[18]. When deep acupuncture at $\mathrm{CV} 2$, the needle tip could follow the path of the gap between the upper edge of the pubic bone and the bladder reaches the prostate capsule. The anatomical capsule of the prostate is closely related to lower urinary tract symptoms. Due to the presence of the capsule, the hyperplastic gland is compressed and bulge toward the urethra and bladder, which aggravates urinary tract obstruction[19-21]. Therefore, we assume that deep acupuncture at $\mathrm{CV} 2$ with needle tip targeting prostate capsule could release the tension of the prostate capsule so that relieve BOO. To determine the efficacy of deep acupuncture at CV2 with needle tip reaches the prostate capsule, we set another deep acupuncture group with the needle tip $10 \mathrm{~mm}$ away from the prostate capsule, and to exclude comfort effect, a sham acupuncture group for placebo control designed.

\section{Objectives $\{7\}$}

This key objective of this study is to assess the efficacy and safety of deep acupuncture at CV2 for men with $\mathrm{BPH}$.

For the primary outcome, we hypothesized that:

1) deep acupuncture at CV2 with needle tip reaching the prostate capsule is more effective in decrease the International Prostate Symptom Score (IPSS) than needle tip $10 \mathrm{~mm}$ away from the capsule; 2) deep acupuncture at CV2 has real effect rather than sham comfort.

For secondary outcomes, we hypothesized that:

1) in improving the quality of life (QoL), increasing the rate of urine flow and reducing residual urine, deep acupuncture reaches the prostate capsule is more effective than needle tip $10 \mathrm{~mm}$ away from the prostate capsule; 2) deep acupuncture at CV2 both needle tip reaches the prostate capsule and needle tip $10 \mathrm{~mm}$ away from the prostate capsule are safety. 


\section{Trial design $\{8\}$}

This is a single-center, randomized, subject-and assessor-blinded, placebo-controlled trial. A total of 120 participants who meet the inclusion criteria will be randomly assigned into deep acupuncture (DA) group 1, deep acupuncture (DA) group 2 and sham acupuncture (SA) group in a 1:1:1 ratio. The trial flowchart is provided in Figure 1.

\section{Methods: Participants, Interventions And Outcomes}

\section{Study setting $\{9\}$}

This study will conduct at Department of Acupuncture, Xiyuan Hospital of China Academy of Chinese Medical Sciences (Beijing, China) from Aug. 2019 to Aug. 2022.

\section{Eligibility criteria $\{10\}$}

Inclusion criteria:

Those who meet all the following criteria can be included:

- Male aged between 50 to 80

- Prostate volume $\geq 24 \mathrm{~cm}^{3}$

- Maximum urinary flow rate $<15 \mathrm{ml} / \mathrm{s}$ and/or urinary retention

- Voluntary and written informed consent

Exclusion criteria:

Those who meet any of the following criteria will be excluded:

- Bladder neck contracture, urethral stricture, urinary calculi, urethritis, urinary tuberculosis, acute prostatitis, acute urinary retention, inflammation of the lower urinary tract (bladder and urethra)

- Serious cardiovascular, liver, kidney or lung diseases

- Prostate/bladder tumour

- Neurological disorders may affect urination

- Uncontrolled or untreatable diabetes/arterial hypertension

- Bleeding disorders

- History of genitourinary surgery

- Received BPH-related treatments within 4 weeks

- Participated in other clinical trials within 4 weeks

- Unsuitable for participating based on researchers' judgment 


\section{Who will take informed consent? $\{26 \mathrm{a}\}$}

Two trained research assistants will be responsible for participants recruitment. Each potentially participant will be fully introduced the purpose, procedure, possible risks of the trial, and be provided written informed consent before allocation. Participants voluntarily decide whether to participate after fully understanding this trial, and they have the right to withdraw from the trial at any time. Research assistants will confidentially store informed consent after qualified participant signed it. At the same time, the subject will receive a copy of signed and dated informed consent form.

\section{Additional consent provisions for collection and use of participant data and biological specimens \{26b\}}

Not applicable. We will not use participant data and biological specimens for ancillary study.

\section{Interventions}

\section{Explanation for the choice of comparators $\{6 \mathrm{~b}\}$}

Based on the result of our pilot study, two experts designed the comparator scheme that acupuncture with different depth to evaluate whether acupuncture to the prostate capsule is more effective than acupuncture $10 \mathrm{~mm}$ away from the prostate capsule, and the sham acupuncture group to exclude sham comfort effect.

\section{Intervention description $\{11 \mathrm{a}\}$}

\section{DA 1}

Subject who is randomized into deep acupuncture (DA) group 1 will receive deep acupuncture at CV2 to the prostate capsule with sterile disposable needle $(0.35 \mathrm{~mm}$ in diameter and $75 \mathrm{~mm}-100 \mathrm{~mm}$ in length, needle length depending on each patient's body figure). Universal brand needle (manufactured by Suzhou Medical Instruments Co., Ltd., China) will be used. According to the World Health Organization Standard Acupuncture Point Locations[18], CV2 is in the lower abdomen, the upper edge of the pubic symphysis and the anterior midline. The first treatment will be performed in the computerized tomography (CT) room of the Department of Radiology.

Operation process: $\otimes$ Before the start of acupuncture treatment, subject will be asked to urinate so as to empty their bladder. Then, subject lies on the examination bed and be pasted a fixed pad on the surface of CV2. खRadiologist measures the supposed needle depth and angle (anatomically, the needle is inserted from the skin of $\mathrm{CV} 2$ and punctured along the gap between the upper edge of the pubic bone and the bladder) on the mid-sagittal level of the subject's lower abdomen under the positioning of the 16-slice spiral CT (GE Optima 680) diagnostic apparatus. \Acupuncturist deep needling at CV2 with sterile disposable needle after disinfect the local skin of CV2, and make the needle tip reaches the prostate capsule refers to the measured depth and angle data. An additional picture file shows this in more detail [see Additional file 1]. खRadiologist observe whether the needle tip reaches the targeted prostate capsule 
by three-dimensional images of CT. 『Acupuncturist puncture CV2 again if the needle tip not reach the prostate capsule. The first treatment not retain the needle.

Data will be carefully managed through a complete digital image transmission and storage management system (PACS/RIS system). The last 9 treatments will be given in the outpatient department of acupuncture based on the data measured under CT and the needle retains for 30 minutes. Each subject's acupuncture sensation and any acupuncture-related response will be carefully recorded. Acupuncture will be performed by an experienced acupuncturist who practice in clinic more than fifteen years to ensure the accuracy and safety.

\section{DA 2}

The acupoint selection, point location, needle tool, and operation process of subject in deep acupuncture (DA) group 2 are the same as DA 1, but the needle tip is about $10 \mathrm{~mm}$ away from the prostate capsule.

\section{SA}

Subject who is randomized into sham acupuncture (SA) group will be punctured with $0.3 \mathrm{~mm} \times 25 \mathrm{~mm}$ sterile disposable needle. After routinely disinfect the local skin of $\mathrm{CV} 2$, acupuncturist will paste the fixed pad on the surface of CV2, and then use acupuncture needle to penetrate the fixed pad, making the needle tip reaches the surface of the skin but does not pierce the skin. Needle retention time is 30 minutes. All of the 10 treatments will be performed in the outpatient department of acupuncture.

\section{Criteria for discontinuing or modifying allocated interventions $\{11 \mathrm{~b}\}$}

- Subject whose condition deteriorated during the study and the intervention should be stopped according to researchers' judgment.

- Subject has poor compliance (treatment frequency $<80 \%$ ), or automatically receive other treatment methods during the trial, such as take medications (e.g., a1-blockers, 5a-reductase inhibitors, antimuscarinics, Chinese patent medicine) that are prohibited by this trial.

- Regardless of the reason, subject can request to withdraw from the trial at any time.

\section{Strategies to improve adherence to interventions $\{11 \mathrm{c}\}$}

To improve subjects' adherence to this trial, two specific assistants are responsible for the connection and arrangement of patients. At predefined time, subjects will be informed to receive examinations and treatments via phone calls or WeChat messages. During the 4 months of the trial, participants will be requested to record their daily urination into a voiding diary to observe their urination changes and to improve trial adherence.

\section{Relevant concomitant care permitted or prohibited during the trial $\{11 \mathrm{~d}\}$}


During the trial, patients will be discouraged from receiving trial-unrelated $\mathrm{BPH}$ treatments and participating in other clinical trials.

\section{Provisions for post-trial care $\{30\}$}

After the end of the study, patients in the sham acupuncture group will be given 10 acupuncture treatments as compensation.

\section{Outcomes $\{12\}$}

\section{Primary outcomes}

Change of the International Prostate Symptom Score (IPSS) before and immediately after the 4 weeks intervention, and at the 8 weeks post-intervention follow-up is defined as primary outcome. The IPSS is a globally recognized quantitative evaluation method for symptoms developed by the American Urological Association (AUA) Measurement Committee in 1991[22]. It contains seven questions on storage and voiding symptoms (incomplete emptying, frequency, intermittency, urgency, weak stream, straining and nocturia). Each sub-themes are scored 0 to 5 , giving a maximum total score of 35 . The severity scoring is as follows: asymptomatic (0 points), mildly symptomatic(1-7points), moderately symptomatic (8-19 points), and severely symptomatic (20-35 points). Subjects will be asked to record their daily voiding symptoms into voiding diary questionnaire[23] from baseline to the end of the trial to assess the changes of IPSS and QoL.

\section{Secondary outcomes}

Change of quality of life (QoL) before and immediately after the 4 weeks intervention, and at the 8 weeks post-intervention follow-up will be defined as one of the secondary outcomes. QoL is the evaluation scale with a 6-point scale (delighted, pleased, mostly satisfied, mixed, dissatisfied, unhappy, terrible) and recommended for BPH patients by the fifth International Advisory Committee of Benign Prostatic Hyperplasia[24].

Other secondary outcomes are the change of the maximum urinary flow rate (Qmax)[25] and post-void residual (PVR). These outcomes will be measured at baseline and 4 weeks after the start of intervention.

\section{Safety evaluation}

\section{Acupuncture safety}

Evaluation of acupuncture safety including broken needles, fainting needles, unbearable acupuncture pain, local hematoma, local infection, local abscess, and other discomforts after acupuncture (e.g., fatigue, palpitations, dizziness, headache, insomnia). The visual analogue scale (VAS), a popular instrument for the measurement of pain intensity, will be used to evaluate the acupuncture pain[26]. The VAS scale ranged between 0 to $100 \mathrm{~mm}$ : no pain ( 0 to $4 \mathrm{~mm}$ ), mild pain ( 5 to $44 \mathrm{~mm}$ ), moderate pain (45 to $74 \mathrm{~mm})$, severe pain $(75$ to $100 \mathrm{~mm})$ [27]. 


\section{Acupuncture tolerance}

The evaluation of acupuncture tolerance includes the discomfort of acupuncture and the acceptance of acupuncture. The greatest degree of discomfort in the treatment process will be evaluated by VAS. Acceptance of patient will be recorded as follows: very difficult to accept (0 points), slightly difficult to accept (1 points), acceptable ( 2 points), easy to accept (3 points), very easy to accept (4 points).

\section{Evaluation point}

Within 5 minutes after the end of the first and sixth treatments, calculate the average score of the two treatments. If one of the VAS values is missing, taking the remaining VAS value as result.

\section{Participant timeline $\{13\}$}

A total of 4 months will be consumed for each subject, including a 4 weeks baseline assessment, a 4 weeks intervention period, and an 8 weeks follow-up period. The baseline screening will be performed before allocation. During the intervention period, patients in each group will receive 10 treatments within 4 weeks with treatment frequency decreasing at 2 weeks intervals ( 3 treatments per week during the first 2 weeks, 2 treatments per week during the last 2 weeks). The following assessment will be completed in 8 weeks after the end of intervention. Figure 2 provides a detailed description of the SPIRIT[28] progression of the trial.

\section{Sample size $\{14\}$}

The sample size estimation was based on the results of the pilot study that the effective rate of experiment groups not less than $80.0 \%$, and the control group not more than $40 \%[17]$. With a significance level of 0.05 (two-sides) and a power of $90 \%$, the calculated sample size in each group is 32 . Assuming a drop-out rate of $20 \%$ or less, a sample size of 40 per group and a total number of 120 subjects will be required. The sample size calculation was performed using the Power Analysis and Sample Size (PASS, NCSS, Kaysville, Utah, USA) version 15.

\section{Recruitment $\{15\}$}

Participants will be recruited through advertisements on the homepage of Xiyuan Hospital which with more than 4 hundred thousand followers and recruitment notices posted at the acupuncture outpatient clinic. Research assistants will in charge of the recruitments of subjects. The potentially eligible subjects who are interested in this trial will be invited to take the screening visit. Before allocation, participants will complete demographic characteristics collection, medical history survey, laboratory examination (routine blood test, urinalysis, liver and kidney function), B-ultrasound (PVR, prostate volume), and uroflowmetry (Qmax) to evaluate their eligibility. Recruitment started in January 2020 and is planned to end in December 2021.

\section{Assignment of interventions: allocation}




\section{Sequence generation $\{16 a\}$}

The random allocation sequence is generated and kept by the staff of the Institute of Clinical Pharmacology using the Statistical Package for the Social Sciences (SPSS, Inc., Chicago, United States) version 22.0 , who is uninvolved in the study operation. A total of 120 patients will be randomly assigned to 3 groups according to a ratio of 1:1:1.

\section{Concealment mechanism \{16b\}}

The concealment of the random allocation sequence uses sequentially numbered, opaque and sealed envelopes. According to the random allocation sequence, cards with assigned groups are placed one by one into sequential, opaque, sealed envelopes. When subject qualified for the baseline assessment, he will get a sequence number, and acupuncturist will obtain the same coded envelope from the envelopekept staff and assign the subject into the appropriate group. Throughout the study, only the acupuncturist is allowed to open sealed envelopes. Subjects, research assistants, envelope-kept staff, outcome assessors will be blinded.

\section{Implementation $\{16 c\}$}

Staff of the Institute of Clinical Pharmacology generate random allocation sequence by SPSS 22.0 software. Research assistants are responsible for recruiting participants. Acupuncturist is in charge of assigning participants to interventions.

\section{Assignment of interventions: Blinding}

\section{Who will be blinded $\{17 a\}$}

Subjects, research assistants, and outcome assessors will be blinded. Because of the particularity of acupuncture clinical trials, acupuncturist should understand the grouping situation so that implement different interventions. To ensure the blindness between subjects, they will be visited separately and be treated in room with curtains. A simple inquiry, whether the subjects had received acupuncture and the sense of acupuncture (sourness, numbness, heaviness, pain, etc.), will be conducted by research assistants within 5 minutes after the end of the first and sixth interventions to assess the success of the blind method.

\section{Procedure for unblinding if needed $\{17 \mathrm{~b}\}$}

- If subject's condition deteriorated during the trial and researchers judge that continued blindness would threaten subject's life safety. The blindness can be broken.

- Emergencies such as serious adverse events (SAEs) or suspicious unintended serious adverse reactions, blindness can be revealed and unblinding is permissible.

\section{Data collection and management}




\section{Plans for assessment and collection of outcomes $\{18 \mathrm{a}\}$}

Before the start of the trial, investigators will be uniformly trained, and each member will clarify their tasks and responsibilities to ensure the data quality and reliability of trial outcomes. Trained research assistants are responsible for the collection of voiding diary questionnaire and clearly filling data into the case report form (CRF). Before CRF submitted to data analysts, data monitor will check the authenticity and reliability of the CRF.

\section{Plans to promote participant retention and complete follow-up $\{18 \mathrm{~b}\}$}

All subjects, whether they are in compliance with the provision of this protocol or not, will be filled data in the CRF by research assistants. All dropped cases will be traced from their most recent assessment and record in detail of the cause and time in CRF. The follow-up visit will take place around 2 days before and after 8 weeks of the end of intervention. Research assistants will invite subject to the hospital for the final visit or score their IPSS and QOL by phone calls.

\section{Data management $\{19\}$}

Data will be checked before locked and sent to the data analysts. The same data will be entered independently by two trained entrees using SPSS 22.0 software, and any inconsistent part shall be modified refer to the original CRF. Double data entry can effectively reduce errors and ensure the accuracy of data. For any question about data, research assistants will carefully check and respond.

\section{Confidentiality \{27\}}

The information of all potential and enrolled participants of this trial will be confidential, and only trial members for the purpose of management will have access to the subject's case records. Chief investigator $(\mathrm{Cl})$, who is also the only unblinded acupuncturist, will not be allowed to access the data until it is completed. The names of the subjects will not appear in any published information or reports on the study. Study researchers will make every effort to protect the privacy of the subject's medical information.

\section{Plans for collection, laboratory evaluation and storage of biological specimens for genetic or molecular analysis in this trial/future use $\{33\}$}

Biological specimens (routine blood test, urinalysis, liver and kidney function) will be collected by nurse of acupuncture department and sent immediately to the inspection department. The inspection reports will be recorded in CRF. The original inspection reports must be consistent with the laboratory examination data recorded in CRF and be traceable.

\section{Statistical methods}

Statistical methods for primary and secondary outcomes $\{20 \mathrm{a}\}$ 
Data will be analyzed by SPSS 22.0 statistical analysis software and performed by a statistician blinded to allocation in the Institute of Clinical Pharmacology of Xiyuan hospital. The normality test or homogeneity of variance test will be performed before data analysis. If the data follow the normal distribution or homogeneity of variance, it will be described by mean \pm standard deviation, intragroup differences will use paired $t$-test, comparisons between groups will use one-way analysis of variation (ANOVA). If data not follow the normal distribution and homogeneity of variance, it will be described by median and range ( $\min$ and $\max$ ), intragroup differences will use non-parametric test, comparisons between groups will use kruskal-wallis $\mathrm{H}$ test. Multiple comparisons posttest will use SNK or Tmhane's T2. To control the increase of false positive errors in multiple comparison, we adjust the significance level of primary outcome by Bonferroni, a set at 0.025 (one-sided) with a power of $90 \%$, a level of $P<0.025$ will be regarded as statistically significant. Secondary outcomes and other comparisons set $\alpha=0.05$ (two-sided), $\beta=0.1, P<0.05$ will be regarded as statistically significant.

\section{Interim analyses $\{21 b\}$}

Not applicable. We will not perform interim analyses.

\section{Methods for additional analyses (e.g. subgroup analyses) $\{20 \mathrm{~b}\}$}

If necessary, subgroup analysis will be performed refer to the severity of the IPSS.

\section{Methods in analysis to handle protocol non-adherence and any statistical methods to handle missing data $\{20 \mathrm{c}\}$}

Data analysis will be based on the per protocol set (PPS). The PPS analysis include participants who have more than $80 \%$ protocol-adherence, i.e., receive at least eight treatments. The last-observationcarried-forward method will be used to process the missing data.

\section{Plans to give access to the full protocol, participant level-data and statistical code $\{31 \mathrm{c}\}$}

The trial protocol and statistical code will be disseminated through open-access journals. The data of participants from this trial can be accessible by contacting the corresponding author.

\section{Oversight and monitoring}

\section{Composition of the coordinating centre and trial steering committee $\{5 \mathrm{~d}\}$}

Beijing Municipal Science and Technology Commission is in charge of the audit to oversee the progress of the study. Staff of Office of Academic Research of Xiyuan hospital will act as data monitor to ensure the trial implementation, documentation and reporting meet ethical and scientific quality requirements.

Composition of the data monitoring committee, its role and reporting structure $\{21 \mathrm{a}\}$ 
Since no adverse events (AEs) reported, data monitoring committee will not be appointed in this trial. Data monitor will be independently with investigators and sponsor, and they have no competing interests.

\section{Adverse event reporting and harms $\{22\}$}

For any $A E$, the time, duration and extent of their occurrence will be documented and investigated. If they are related to the intervention, symptoms will be closely followed until they disappear. SAEs related to the study will be immediately reported to $\mathrm{Cl}$, sponsor, and Ethics Committee of Xiyuan Hospital within 24 hours.

\section{Frequency and plans for auditing trial conduct $\{23\}$}

Every 12 months, all CRF forms will be regularly audited by the supervisors set up by Beijing Municipal Science and Technology Commission. This process will be independent from investigators and the sponsor.

\section{Plans for communicating important protocol amendments to relevant parties (e.g. trial participants, ethical committees) $\{25\}$}

Important protocol amendments (e.g., inclusion/exclusion criteria, outcome measure methods, data analysis methods) will submit to the Ethics Committee of Xiyuan Hospital and modifications will subsequently be described in trial reports.

\section{Dissemination plans $\{31$ a $\}$}

Investigators and sponsor will communicate the trial results to participants, healthcare professionals, the public, and other relevant groups through open access journals and conferences. The trial permits unrestricted use and distribution.

\section{Discussion}

The current interventions for BPH mainly includes watchful waiting, pharmacotherapy, and surgical treatment[29]. Acupuncture as an alternative treatment for men with BPH has been widely evaluated recent years $[16,30]$. This study is designed to provide an evidence-based recommendation on whether deep acupuncture at CV2 is a valuable alternative treatment for men with bothersome BPH/LUTS. To our knowledge, this is the first randomized, placebo-controlled trial to investigate the efficacy of deep acupuncture at CV2. We innovatively combine the theory of acupuncture with anatomy into clinical practice, which is expected to offer a better understanding of the underlying mechanism of acupuncture at $\mathrm{CV} 2$. Subject in DA1 and DA2 will be measured the needle depth and angle under CT to investigate the safety of deep acupuncture at CV2. Furthermore, we expected to summarize the correct operation method through these CT images. 
The practice of sham acupuncture is a challenge part of this study. Participants in sham acupuncture group will receive false needle, with the needle tip piercing the fixed pad to reach the skin surface causing subjects a sensation of pain, but the needle tip not pierce the skin to minimize the therapeutic effect. Considering the success of the blindness, subjects allocated to different groups will be visited separately and treated in room with curtains. After the first and sixth interventions, subjects will be asked if they knew they were receiving acupuncture and the sensation of acupuncture to determine the success of blindness. To compensate participants in the sham acupuncture group, they will be given 10 times acupuncture treatments after the end of the study. All subjects in three groups will be pasted fixed pad on the surface of $\mathrm{CV} 2$ before each treatment to minimize the interference factors and to ensure the consistency of the trial. This trial will analysis the changes of IPSS, QoL, Qmax, and PVR from baseline to the end of 4 weeks intervention. We will follow-up the IPSS and QoL 8 weeks after the end of intervention to assess the long-term effectiveness. The results of the study may contribute to the development of new treatment approaches for male with BPH or the associated LUTS.

\section{Trial Status}

The trial commenced in January 2020. The approval of the ethics committee was given in 18 January 2020. Recruitment started in January 2020 and is planned to end in December 2022, with 120 patients randomized. The current protocol version is 2.0, dated 16 December 2019. This trial is in the recruiting phase at the time of manuscript submission.

\section{Abbreviations}

BPH: Benign prostate hyperplasia

BOO: Bladder outlet obstruction

LUTS: Lower urinary tract symptoms

IPSS: International prostate symptom score

QoL: Quality of life

CT: Computerized tomography

Qmax: maximum urinary flow rate

PVR: Post-void residual

SAE: Serious adverse event

CRF: Case report form 
Cl: Chief investigator

ANOVA: Analysis of variation

PPS: Per protocol set

AE: Adverse event

GCP: Good clinical practice

\section{Declarations}

\section{Acknowledgements}

We would like to thank all the investigators and participants involved in the study.

\section{Authors' contributions $\{31 \mathrm{~b}\}$}

$\mathrm{YHL}$ is the $\mathrm{Cl}$, he conceived the study, developed the procedures and protocol, performed the intervention, and responsible for the administration of the study. HT drafted the protocol manuscript. HR and YLM contributed to the measurement and location of the needle depth and angle under CT. YHL and JHF developed the control group plan. HT and BYL assist its implementation and responsible for participants recruitment. FL designed the statistical analysis and the randomization process. XZS contributed to the examination of prostate volume and PVR by B-ultrasound. All authors have read and approved the final manuscript.

\section{Funding $\{4\}$}

The study was funded by Beijing Science and Technology Planning Project (Z191100006619055) of Beijing Municipal Science and Technology Commission. The raw data obtained will be processed independently. It will not influence the unbiased and scientific character of the publication. The trial result will not be influenced by any agency or anyone in any way.

\section{Availability of data and materials $\{29\}$}

Trial investigators have access to the final trial dataset. $\mathrm{Cl}$ and statisticians do not allow access to data until data collection is complete.

\section{Ethics approval and consent to participate $\{24\}$}

The final revised version of the study protocol (version 2, dated 16 December 2019) and the written informed consent used in the study were assessed and approved by the Ethics Committee of Xiyuan Hospital of China Academy of Chinese Medical Sciences (2019XLA063-2). This trial will be performed in accordance to the World Medical Association Declaration of Helsinki, the guidelines for Good Clinical 
Practice (GCP), and relevant Chinese clinical trial research norms and regulations. Written, informed consent will be obtained from every participant prior to registration.

\section{Consent for publication $\{32\}$}

The authors state they will be willing to provide a model consent form on request.

\section{Competing interests $\{28\}$}

The authors declare that they have no competing interests.

\section{Authors' information (optional)}

${ }^{1}$ Department of Acupuncture, Xiyuan Hospital of China Academy of Chinese Medical Sciences, Beijing, China. No.1 Xiyuan Playground, Haidian District, Beijing 100091, China.

${ }^{2}$ Department of Radiology, Xiyuan Hospital of China Academy of Chinese Medical Sciences, Beijing, China. No.1 Xiyuan Playground, Haidian District, Beijing 100091, China.

${ }^{3}$ Office of Academic Research, Xiyuan Hospital of China Academy of Chinese Medical Sciences, Beijing, China. No.1 Xiyuan Playground, Haidian District, Beijing 100091, China.

${ }^{4}$ Institute of Clinical Pharmacology, Xiyuan Hospital of China Academy of Chinese Medical Sciences, Beijing, China. No.1 Xiyuan Playground, Haidian District, Beijing 100091, China.

${ }^{5}$ Department of B-Ultrasound, Xiyuan Hospital of China Academy of Chinese Medical Sciences, Beijing, China. No.1 Xiyuan Playground, Haidian District, Beijing 100091, China.

\section{References}

1. McVary KT, Roehrborn CG, Avins AL, Barry MJ, Bruskewitz RC, Donnell RF, et al. Update on AUA Guideline on the Management of Benign Prostatic Hyperplasia. J Urology. 2011;185(5):1793-803.

2. Gravas S, Cornu J, Gacci M, Gratzke C, Herrmann T, Mamoulakis C, et al. EAU Guidelines on management of non-neurogenic male lower urinary tract symptoms (LUTS), incl. benign prostatic obstruction (BPO). European Association of Urology. 2019. https://uroweb.org/guideline/treatmentof-non-neurogenic-male-luts/ Accessed 19 September 2019.

3. Madersbacher S, Sampson N, Culig Z. Pathophysiology of Benign Prostatic Hyperplasia and Benign Prostatic Enlargement: A Mini-Review. Gerontology. 2019;65(5):458-64.

4. Thorpe A, Neal D. Benign prostatic hyperplasia. Lancet. 2003;361(9366):1359-67. doi:10.1016/S0140-6736(03)13073-5.

5. Berry SJ, Coffey DS, Walsh PC, Ewing LL. The development of human benign prostatic hyperplasia with age. The Journal of urology. 1984;132(3):474. 
6. Egan KB. The Epidemiology of Benign Prostatic Hyperplasia Associated with Lower Urinary Tract Symptoms. Urol Clin N Am. 2016;43(3):289-97.

7. Yu P-L, Zheng H, Su H-X, Zuo T, Duan C-B, Gao F-K, et al. Prevalence of prostatic hyperplasia and its relative factors in six cities of China. Chinese Journal of Epidemiology. 2000;04:36-9.

8. Vuichoud C, Loughlin KR. Benign prostatic hyperplasia: epidemiology, economics and evaluation. Can J Urol. 2015;22(Suppl 1):1-6.

9. Kim EH, Larson JA, Andriole GL. Management of Benign Prostatic Hyperplasia. Annu Rev Med. 2016; 67:137 - 51. doi: 10.1146/annurev-med-063014-123902.

10. Miernik A, Gratzke C. Current Treatment for Benign Prostatic Hyperplasia. Deutsches Ärzteblatt international. 2020;117(49):843-54. doi:10.3238/arztebl.2020.0843.

11. Chughtai B, Forde JC, Thomas DDM, Laor L, Hossack T, Woo HH, et al. Benign prostatic hyperplasia. Nat Rev Dis Primers. 2016;2(1). doi:10.1038/nrdp.2016.31.

12. Foster HE, Barry MJ, Dahm P, Gandhi MC, Kaplan SA, Kohler TS, et al. Surgical Management of Lower Urinary Tract Symptoms Attributed to Benign Prostatic Hyperplasia: AUA Guideline. J Urology. 2018;200(3):612-9. doi:10.1016/j.juro.2018.05.048.

13. Traish AM, Melcangi RC, Bortolato M, Garcia-Segura LM, Zitzmann M. Adverse effects of 5areductase inhibitors: What do we know, don't know, and need to know? Reviews in Endocrine Metabolic Disorders. 2015;16(3):177-98. doi:10.1007/s11154-015-9319-y.

14. Nguyen D, Marchese M, Cone EB, Paciotti M, Basaria S, Bhojani N, et al. Investigation of Suicidality and Psychological Adverse Events in Patients Treated With Finasteride. Jama Dermatol. 2021;157(1):35. doi:10.3238/arztebl.2012.0379.

15. Welliver C, Essa A. Sexual Side Effects of Medical and Surgical Benign Prostatic Hyperplasia Treatments. Urol Clin N Am. 2016;43(3):393-404.

16. Zhang W, Ma L, Bauer BA, Liu Z, Lu Y. Acupuncture for benign prostatic hyperplasia: A systematic review and meta-analysis. Plos One. 2017;12(4):e174586.

17. Lu Y-H, Yan X-H. Thirty-three cases of benign prostatic hyperplasia treated with deep needling at Qugu point (CV2). Chinese Journal of Acupuncture Moxibustion. 2019;39(06):613-4.

18. Huang L-X. The World Health Organization Western Pacific Region, World Health Organization Standard Acupuncture Point Locations. 2: Beijing, People's Medical Publishing House; 2010. p. 212.

19. Ittmann M. Anatomy and Histology of the Human and Murine Prostate. Csh Perspect Med. 2018;8(5):a30346. doi:10.1101/cshperspect.a030346.

20. Na Y-Q, Ye Z-Q, Sun Y-H, Sun G, Huan J, Kong C-Z, et al. Guidelines for Diagnosis and Treatment of Urological Diseases in China. Beijing: People's Health Publishing; 2014. pp. 245-66.

21. Kitzing YX, Prando A, Varol C, Karczmar GS, Maclean F, Oto A. Benign Conditions That Mimic Prostate Carcinoma: MR Imaging Features with Histopathologic Correlation1. Radiographics. 2016;36(1):162-75. doi:10.1148/rg.2016150030. 
22. Barry MJ, Fowler FJ, O'Leary MP, Bruskewitz RC, Holtgrewe HL, Mebust WK, et al. The American Urological Association Symptom Index for Benign Prostatic Hyperplasia. J Urology. 2017;197(2S).

23. Bryan NP, Chapple CR. Frequency Volume Charts in the Assessment and Evaluation of Treatment: How Should We Use Them? Eur Urol. 2004;46(5):636-40.

24. Abrams P, Chapple C, Khoury S, Roehrborn C, de la Rosette J. Evaluation and Treatment of Lower Urinary Tract Symptoms in Older Men. J Urology. 2009;181(4):1779-87. doi:10.1148/rg.2016150030.

25. Kranse R, van Mastrigt R. Causes for variability in repeated pressure-flow measurements. Urology. 2003;61(5):930-4.

26. Reed MD, Van Nostran W. Assessing pain intensity with the visual analog scale: A plea for uniformity. The Journal of Clinical Pharmacology. 2014;54(3):241-4. doi:10.1002/jcph.250.

27. Jensen M. Interpretation of visual analog scale ratings and change scores: a reanalysis of two clinical trials of postoperative pain. The Journal of Pain. 2003;4(7):407-14. doi:10.1016/s15265900(03)00716-8.

28. Chan A-W, Tetzlaff JM, Altman DG, Laupacis A, Gøtzsche PC, Krleža-Jerić K. et,al. SPIRIT 2013 Statement: Defining standard protocol items for clinical trials. Ann Intern Med. 2013;158(3):200-7.

29. Gravas S, de la Rosette JJ. Investigational therapies targeted to the treatment of benign prostatic hyperplasia. Expert Opin Inv Drug. 2013;22(3):357-68. doi:10.1517/13543784.2013.761973.

30. Zhang T, Xun Y, Li B, Wang G, Wang L, Jia L, et al. Effect of fire needle therapy on mild-moderate benign prostatic hyperplasia. Medicine. 2020;99(21):e20376. doi:10.1097/MD.0000000000020376.

\section{Figures}




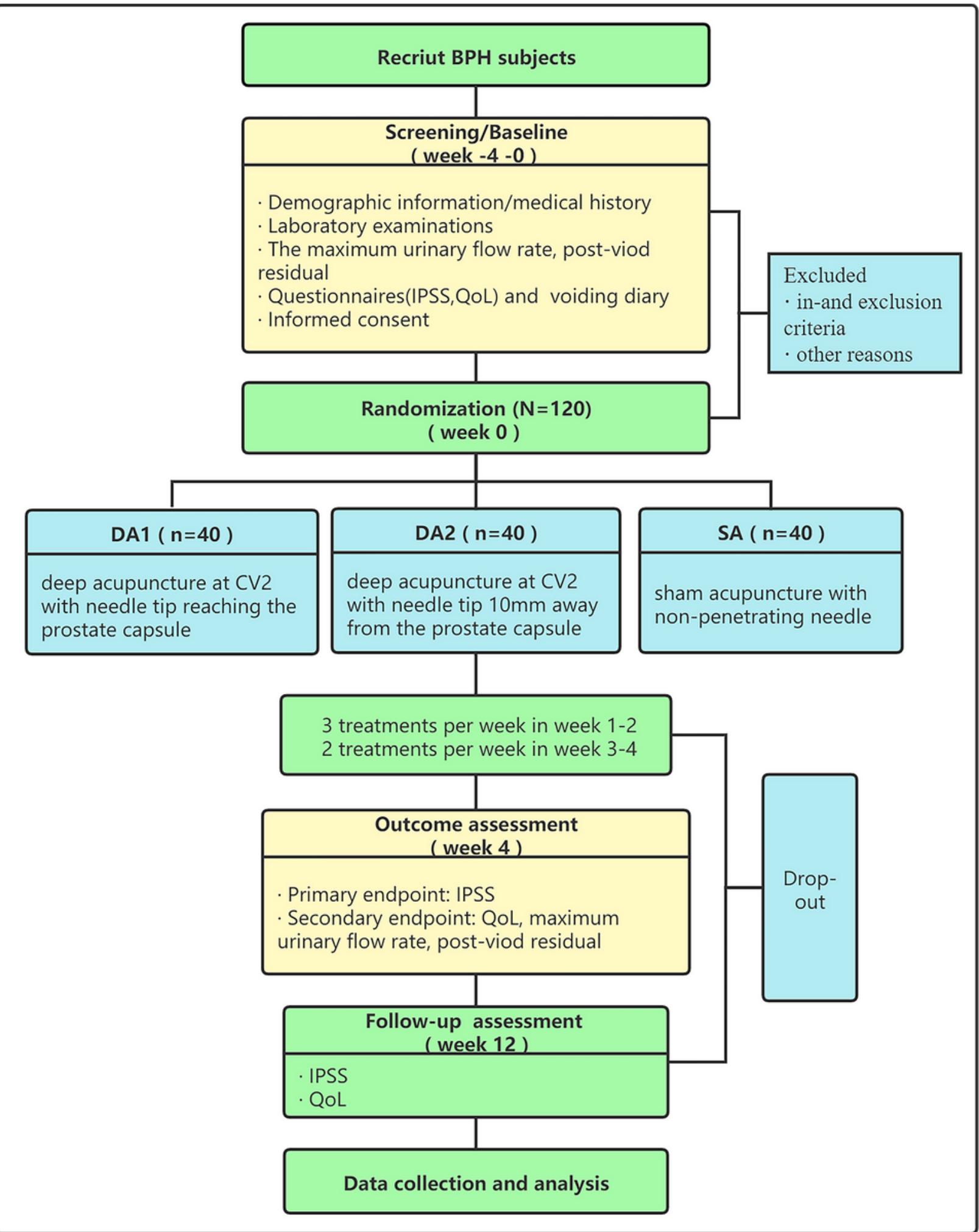

\section{Figure 1}

Trial flowchart. BPH=benign prostatic hyperplasia, IPSS=International Prostate Symptom Score, QoL=quality of life, $D A=$ deep acupuncture, $S A=$ sham acupuncture, $C V 2=Q u g u$ point (PNG 791KB pixels: $1678 * 2100)$ 


\begin{tabular}{|c|c|c|c|c|c|c|c|}
\hline \multirow[b]{3}{*}{ TIMEPOINT (week) } & \multicolumn{7}{|c|}{ STUDY PERIOD } \\
\hline & \multirow{2}{*}{$\begin{array}{c}\text { Enrolment } \\
-4\end{array}$} & \multirow{2}{*}{$\begin{array}{c}\text { Allocation } \\
0\end{array}$} & \multicolumn{4}{|c|}{ Post-allocation } & \multirow{2}{*}{$\frac{\text { Follow-up }}{12}$} \\
\hline & & & 1 & 2 & 3 & 4 & \\
\hline \multicolumn{8}{|l|}{ ENROLMENT: } \\
\hline \multirow[b]{2}{*}{ Laboratory examination } & $X$ & $X$ & & & & & \\
\hline & $X$ & & & & & & \\
\hline Informed consent & $X$ & & & & & & \\
\hline Allocation & & $X$ & & & & & \\
\hline \multicolumn{8}{|l|}{ INTERVENTIONS: } \\
\hline \multicolumn{8}{|l|}{ Deep acupuncture group1 } \\
\hline \multicolumn{8}{|l|}{ Deep acupuncture group2 } \\
\hline \multicolumn{8}{|l|}{ Sham acupuncture group } \\
\hline \multicolumn{8}{|l|}{ ASSESSMENTS: } \\
\hline \multirow[t]{3}{*}{$\begin{array}{r}\text { International Prostate } \\
\text { Symptom Score }\end{array}$} & $X$ & & & & & $X$ & $X$ \\
\hline & $X$ & & & & & $X$ & $X$ \\
\hline & $X$ & & & & & $X$ & \\
\hline Post-void residual & $X$ & & & & & $X$ & \\
\hline Prostate volume & $X$ & & & & & $\mathrm{X}$ & \\
\hline Voiding diary & $X$ & $X$ & $X$ & $\mathrm{X}$ & $X$ & $X$ & $X$ \\
\hline Acupuncture safety & & & $X$ & & $X$ & & \\
\hline Acupuncture tolerance & & & $X$ & & $X$ & & \\
\hline Adverse events & & & $X$ & $X$ & $X$ & $X$ & \\
\hline
\end{tabular}

Figure 2

SPIRIT figure of schedule of enrolment, interventions, and assessments. (PNG 532KB pixels: 1678*2100)

\section{Supplementary Files}

This is a list of supplementary files associated with this preprint. Click to download. 
- Additionalfile1.pdf

Page 20/20 